\title{
MHD boundary layer flow over a non-linear stretching boundary with suction and injection
}

\author{
Ramesh B. Kudenatti ${ }^{\text {a,*}}$, Shreenivas R. Kirsur ${ }^{\text {b }}$, L.N. Achala ${ }^{\text {b }}$, N.M. Bujurke ${ }^{\text {c }}$ \\ ${ }^{a}$ Department of Mathematics, Bangalore University, Bangalore 560 001, India \\ ${ }^{\mathrm{b}}$ P.G. Department of Mathematics and Research Centre in Applied Mathematics, MES College, Malleswaram, Bangalore 560 003, India

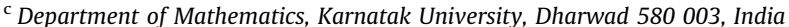

\section{A R T I C L E I N F O}

\section{Article history:}

Received 19 September 2012

Received in revised form

9 October 2012

Accepted 7 November 2012

Available online 20 November 2012

Keywords:

MHD

Boundary layer equations

Falkner-Skan equation

Exact solution

Asymptotic solution

\begin{abstract}
A B S T R A C T
In this paper, we give an exact solution to the most celebrated magnetohydrodynamic Falkner-Skan equation. The equation governs the two-dimensional laminar boundary layer flow of a viscous, incompressible and electrically conducting fluid over a semi-infinite flat plate in the presence of magnetic field. Similarity transformations are used to convert the governing coupled non-linear partial differential equations into a highly non-linear ordinary differential equation with boundary conditions. An exact analytical solution is obtained for certain parameters which is then modified and generalized to give an exact solution to all other involved parameters. The results thus obtained are compared with that of direct numerical solutions, which agree well up to desired accuracy. The MHD Falkner-Skan equation exhibits the upper and lower branch solutions that reveal a very interesting velocity profiles for a set of parameters. Results are presented in the form of velocity profiles and skin friction for various values of physical parameters and are discussed in detail.
\end{abstract}

(c) 2012 Elsevier Ltd. All rights reserved.

\section{Introduction}

We consider a steady MHD two-dimensional boundary layer flow of a viscous and incompressible electrically conducting fluid in the presence of the magnetic field which is obeyed by the MHD Falkner-Skan equation

$f^{\prime \prime \prime}(\eta)+f(\eta) f^{\prime \prime}(\eta)+\beta\left(1-f^{\prime 2}(\eta)\right)-M^{2}\left(f^{\prime}(\eta)-1\right)=0$,

with boundary conditions

$f(0)=\alpha, \quad f^{\prime}(0)=-\lambda, \quad f^{\prime}(+\infty)=1$,

where $\beta$ is stream-wise pressure gradient parameter and $M$ is the magnetic (Hartmann) number. See Appendix for the derivation of MHD Falkner-Skan equation. Parameter $\lambda$ represents the stretch of the boundary, and $\alpha$ is the suction or injection parameter. For $M=\alpha=0=\lambda$, the above system reduces to the classical FalknerSkan equation. Interestingly, we see that $f(\eta)=\eta+\alpha$ is an exact solution of system (1) and (2) for $\lambda=-1$, then velocity gradient becomes zero which demarcates the solution nature across boundary layer flow. We note here that, though the above governing ordinary differential equation is not stiff, it seems it is more difficult to solve analytically because of its high nonlinearity. We discuss some of the significant features of solution of the above system in the absence of magnetic field. Behavior

\footnotetext{
* Corresponding author. Tel.: +91 80 22961424; fax: +91 8022219714

E-mail address: ramesh@bub.ernet.in (R.B. Kudenatti).
}

of the boundary layer flow over the flat plate has considerable engineering applications, such as drawing of plastic films, spinning of metals, insulating materials, fine-fiber matts, etc. In the process, the plate while moving in its own plane may induce a motion in the neighboring fluid or fluid may move independently parallel to it. This would have great impact on the final products. On the other hand, the boundary layer flow over stretching surface with porous media has been studied in the past due to many engineering applications in petroleum and geothermal industry, food processing, extraction of geothermal energy, etc. Because of these applications, many investigators studied the above problem in different contexts (Rajagopal et al. [1], Hsu et al. [2], Olagunju [3], Anabtawi and Khuri [4], Vera and Valencia [5], etc.).

Numerous papers are available in the literature for the numerical solution of the above system for different values of $\beta$ with different boundary cases (Hartree [6], Stewartson [7], Libby and Liu [8], etc.). They have proved the existence of multiple solutions of the above system for some range of pressure gradient parameter $\beta$. The numerical computations were refined by Riley and Weidman [9] for the Falkner-Skan equation with $\alpha=0$ over the range of parametric values of $\beta$ presuming the overshoot velocity profiles have physical meanings. Surprisingly, their computations revealed that, when $\lambda>0$, and an appropriate choice of $\beta$, the system admits a unique solution in the range $0.14 \leq \beta \leq 0.5$, dual solutions for $0.5 \leq \beta \leq 1$, and even triple solutions for $0<\beta \leq 0.14$. The physical mechanism underlying this behavior was discussed in detail by Riley and Weidman [9]. 
All the above mentioned solutions to the Falkner-Skan equation are given by the numerical methods in different context. To the authors knowledge, no closed form solution has been found in the literature because of its robust non-linearity and an infinite interval. As pointed out by Liao [10], the perturbation techniques have been applied to most of the boundary layer problems which involve one or more small parameters. Using this technique in higher order terms, Afzal and Luthra [11] have predicted the skin friction $f^{\prime \prime}(0)$ for different values of $\beta$. Furthermore, Fang and Zhang [12] and Afzal [13] have reported that the Falkner-Skan problem (1) and (2) admits a closed-form analytical solution for $\beta=-1$ apparently this solution is a combination of solutions of Yang and Chien [14] with $\lambda=0$ and Riley and Weidman [9] with $\alpha=0$. Twice integration of (1) and (2) with $\beta=-1$ gives the Riccati type equation

$f^{\prime}(\eta)+\frac{f^{2}(\eta)}{2}=\frac{\eta^{2}}{2}+\delta \eta-\alpha \lambda \eta-\lambda+\frac{\alpha^{2}}{2}$,

where $\delta=f^{\prime \prime}(0)$. The solution of (3) is given by

$$
\begin{aligned}
f(\eta)= & \eta \delta-\alpha \lambda \\
& +\frac{(\alpha(1+\lambda)-\delta) e^{-\left(\left(\eta^{2} / 2\right)+(\delta-\alpha \lambda) \eta\right)}}{1+(\alpha(1+\lambda)-\delta) \sqrt{\frac{\pi}{8}} e^{\frac{1}{2}(\delta-\alpha \lambda)^{2}}\left(\operatorname{erf}\left(\frac{\eta+\delta-\alpha \lambda}{\sqrt{2}}\right)-\operatorname{erf}\left(\frac{\delta-\alpha \lambda}{\sqrt{2}}\right)\right)},
\end{aligned}
$$

provided $\delta=\alpha \lambda \pm \sqrt{-2(1+\lambda)+\alpha^{2}}$. Differentiation of (4) provides the velocity profiles for various of values of $\alpha$ and $\lambda$. The gradient of axial velocity at the wall can be obtained

$f^{\prime \prime}(0)=\delta=\alpha \lambda \pm \sqrt{-2(1+\lambda)+\alpha^{2}}$.

From Eq. (5), it follows that, the Falkner-Skan equation exhibits dual solutions for $-2(1+\lambda)+\alpha^{2} \geq 0$, and no solution for $-2(1+\lambda)+$ $\alpha^{2}<0$. Exploiting analytical structure of solution (4) for $\beta=-1$, Fang and Zhang [12] have divided the velocity profiles as the upper branch solution when $\alpha<0$, and as the lower branch solution for $\alpha>0$ when wall stretching parameter $\lambda$ is held constant. So, rigorously speaking, the closed form solution (4) is the only available solution for the Falkner-Skan equation for $\beta=-1$ but fail to do so for other values of pressure gradient parameter $\beta$. Therefore, it is worthwhile to develop a new analytical techniques for all possible values of $\beta$ which seem to have physical meanings. In this category, Liao's [10] homotopy analysis method gives a uniformly valid, and convergent assured series solution for the Falkner-Skan family of equations. This method naturally embeds the analytical continuation techniques in its base function that ensures the uniformly valid solution. Notice that, this method involves moderately higher order approximations in order to obtain a reasonable accuracy for derived quantities such as skin friction $\left(f^{\prime \prime}(0)\right)$, velocity profiles $\left(f^{\prime}(\eta)\right)$, etc. Exploring this almost uniformly valid solution method, many investigators have applied to non-linear differential equations arising in two-dimensional boundary layer flow (Yao [15], Yao and Chen [16], Raftari and Vajravelu [17] and so on).

All the analytical solution of the Falkner-Skan equation for $\beta=-1$ that have been reported in the past are for different boundary conditions (Yang and Chein [14], Riley and Weidman [9], Fang and Zhang [12], and Afzal [13]). Rewriting, for convenience, Eq. (4) as

$f(\eta)=\Lambda+\eta-\frac{\Lambda-\alpha}{G(\eta)}$,

where $\Lambda=\delta-\alpha \lambda$, and the new stream-function $G(\eta)$ shall be defined in Section 2. Form (6) closely resembles with that of a single hump solution of the Burger's equation (Sachdev [18]). Using similar function like (6), Sachdev et al. [19,20] gave an exact solution of the generalized Burgers equation for all involved parameters via Euler-Painleve transcendents which characterize the analytic properties of the generalized Burgers equation. Using the above concept, Sachdev et al. [21] gave an exact solution of the Falkner-Skan equation for all possible values of pressure gradient parameter $\beta$. Their method embeds an exact analytical solution (4) of the Falkner-Skan equation for $\beta=-1$ (boundary conditions (2) with $\alpha=0$ ) as a special case, and recovers all earlier solutions. Recently, Kudenatti [22] successfully adopted the above technique to solve the Falkner-Skan equation modified by the reference velocity which is composed of free-velocity and the velocity at the wall, and gave an exact solution of the system when $\alpha=0$ for all values of pressure gradient parameter.

Along with usual boundary layer applications, magnetohydrodynamic (MHD) flows of viscous fluids have been performed due to large number of applications in industries, for instance, MHD power generation, MHD flow meters and MHD pumps. In polymer industry, when cooling of continuous strips or filaments through quiescent fluid takes place, these strips are often get stretched, thus, this cooling can be effectively managed by passing the stretched strips through a magnetic field so that final products would have desired characteristics. This cooling largely depends on the applied magnetic field. The study of effects of MHD applications can be found in other applications related to stretching surfaces. In general, the magnetic fields have the stabilizing capacity of the boundary layer flow. Because of these significant applications, many mathematical models have been proposed to explain the behaviors of the viscous MHD boundary layer flow under different conditions. Rashidi and Erfani [23] have investigated MHD boundary layer flow in the porous medium using a combination of the differential transform method and Pade' approximants, and discussed the influence of all parameters on the velocity and temperature profiles.

In the present study, significant advances in obtaining an exact solution of the Falkner-Skan problem (1) and (2) for all values of $\beta$ and Hartman number $M$ have been achieved through similar approach pioneered by Sachdev et al. [21]. We follow the work of Sachdev et al. [21] and Kudenatti [22] to obtain the exact solution of the problem for all $\beta$ and $M$, and for ease of completeness and to make the paper self-contained some derivations have been given again with regard to the present problem. Our exact solution exhibits a very interesting solution branches which are not found in most of numerical investigations. Although the exact solution of the problem is, however, not possible for all parameters except for $\beta=-1$, these multiple solutions have not been reported. The variety of possible mechanisms for multiple solutions has led to the desire and robust mathematical analysis of the governing equation. As a result, the inherent of a parameter (see Eq. (13) below) in our solution method makes these solutions to exist. In the process, we first give an exact solution to the problem in discussion, and then we shall discuss the nature of solutions in detail.

Rest of the paper is organized as follows. In Section 2, we give an exact solution of the MHD Falkner-Skan Eq. (1) with boundary conditions (2) for general values of $\beta$ and $M$. The modified form (6) has been utilized for this purpose. Various physical parameters in the form of velocity profiles and skin friction coefficient also have been discussed in this section. Asymptotic solution of problem for large $\eta$ has been given in Section 3, and solution nature has also been discussed briefly. In Section 4 we discuss the important results of the method. Final section summarizes the importance of the method and its possible generalization to other boundary layer equations.

\section{Exact solution for general $\beta$ and $M$}

In this section, we give an exact solution to (1) and (2). Notice from (4) that, we have already obtained a closed form solution to (1) and (2) for $\beta=-1$ and $M=0$ which is rewritten in the form of (6). Now, the essence is to transfer expression (6) into another non-linear ordinary differential equation in $G(\eta)$ which contains the parameters $\beta$ and $M$. To do so, substituting (6) into (1), we get 
the following equation:

$$
\begin{aligned}
G^{2} G^{\prime \prime \prime} & -G G^{\prime \prime}(6 G-(\alpha(1+\lambda)-\delta)-(\eta+\delta-\alpha \lambda) G) \\
& -G^{2} G^{\prime}\left(2 \beta+M^{2}\right)-G^{\prime 2}((\alpha(1+\lambda)-\delta)(2-\beta) \\
& +2(\eta+\delta-\alpha \lambda) G)+6 G^{\prime 3}=0,
\end{aligned}
$$

and the boundary conditions (2) become

$G(0)=1, \quad G^{\prime}(0)=\frac{1+\lambda}{\alpha(1+\lambda)-\delta}, \quad G(+\infty)=\infty$,

where $G=G(\eta)$. It readily follows from (6) that, an exact analytical solution of ( 7 ) and ( 8 ) for $\beta=-1$ and $M=0$ is given by

$$
\begin{aligned}
G(\eta)= & e^{\left(\eta^{2} / 2+(\delta-\alpha \lambda) \eta\right)}+\sqrt{\frac{\pi}{8}}(\alpha(1+\lambda)-\delta) e^{\frac{1}{2}(\eta+\delta-\alpha \lambda)^{2}} \\
& \times\left(\operatorname{erf}\left(\frac{\eta+\delta-\alpha \lambda}{\sqrt{2}}\right)-\operatorname{erf}\left(\frac{\delta-\alpha \lambda}{\sqrt{2}}\right)\right),
\end{aligned}
$$

where erf( ) is the error function. The error and exponential functions in (9) are entire functions that can be expanded using Taylor series at the onset of the boundary layer which have an infinite radius of convergence. Thus, the series representation of solution (9) becomes the main clue for the further similar analysis for general $\beta$ and $M$. Expecting the similar series representation, it is natural to express the stream-function $G(\eta)$ as

$G(\eta)=\sum_{n=0}^{\infty} a_{n} \eta^{n}$

for general $\beta$ and $M$. For obtaining the coefficients $a_{n}$, substituting (10) into (7) and equating the coefficients of $\eta^{n}$ to zero, we get

$$
\begin{aligned}
a_{0}= & , \quad a_{1}=\frac{1+\lambda}{\alpha(1+\lambda)-\delta}, \\
a_{3}= & \frac{1}{6(\alpha-\delta+\alpha \lambda)^{3}}(-1-\lambda)\left(6-M^{2} \delta^{2}-\beta \delta^{2}+12 \lambda+\beta \delta^{2} \lambda+6 \lambda^{2}\right. \\
& \left.+2 \alpha \delta(1+\lambda)\left(1+M^{2}+\beta-\beta \lambda\right)-\alpha^{2}(1+\lambda)^{2}\left(2+M^{2}+\beta-\beta \lambda\right)\right) \\
& -2(\alpha-\delta+\alpha \lambda)^{2}(-6(1+\lambda)+\alpha(\alpha-\delta+\alpha \lambda)) a_{2}, \\
a_{4}= & -\frac{15017}{8}\left(\frac{1}{(\alpha-\delta+\alpha \lambda)^{4}}\right)(1+\lambda)\left(\alpha ^ { 3 } \delta \left(-4-3 M^{2}\right.\right. \\
& +3 \beta(-1+\lambda)(1+\lambda)^{2}+\alpha^{4}(1+\lambda)^{3}\left(2+M^{2}+\beta-\beta \lambda\right) \\
& +\alpha \delta\left(-4(-4+\lambda)(1+\lambda)^{2}+M^{2}\left(-\delta^{2}+12(1+\lambda)^{2}\right)\right. \\
& \left.+\beta\left(\delta^{2}(-1+\lambda)-8(-2+\lambda)(1+\lambda)^{2}\right)\right)+\alpha^{2}(1+\lambda)\left(3 M ^ { 2 } \left(\delta^{2}\right.\right. \\
& \left.-2(1+\lambda)^{2}\right)+2\left(\delta^{2}+(-8+\lambda)(1+\lambda)^{2}\right)+\beta\left(-3 \delta^{2}(-1+\lambda)\right. \\
& \left.\left.+4(-2+\lambda)(1+\lambda)^{2}\right)\right)+2(1+\lambda)\left(12-3 M^{2} \delta^{2}+2 \beta \delta^{2}(-2+\lambda)\right. \\
& \left.\left.+\lambda\left(\delta^{2}+12(2+\lambda)\right)\right)\right)-\frac{1}{(\alpha-\delta+\alpha \lambda)^{2}}\left(2 \left(12+M^{2} \delta^{2}-2 \alpha^{3} \delta(1+\lambda)\right.\right. \\
& +\alpha^{4}(1+\lambda)^{2}-2 \alpha \delta(1+\lambda)\left(-4+M^{2}+\lambda-2 \beta \lambda\right)+\lambda\left((1-2 \beta) \delta^{2}\right. \\
& +12(2+\lambda))+\alpha^{2}\left(\delta^{2}+M^{2}(1+\lambda)^{2}\right. \\
& \left.\left.\left.\left.-(1+\lambda)^{2}(8-\lambda+2 \beta \lambda)\right)\right)\right) a_{2}-24 a_{2}^{2}\right), \quad \text { etc. },
\end{aligned}
$$

and in general the recurrence relation is

$$
\begin{aligned}
& a_{n+3}=\frac{-1}{(n+1)(n+2)(n+3)} \\
& \left(\sum_{m=0}^{n-1} \sum_{k=0}^{n-m}(m+1)(m+2)(m+3) a_{k} a_{n-m-k} a_{m+3}\right. \\
& \quad+\sum_{m=0}^{n}(-(\delta-\alpha(1+\lambda)))(m+1)\left((m+2) a_{n-m} a_{m+2}\right. \\
& \left.-(2-\beta)(n-m+1) a_{m+1} a_{n-m+1}\right) \\
& -\sum_{m=0}^{n} \sum_{k=0}^{m}(k+1)(m-k+1)\left(2(\delta-\alpha \lambda) a_{n-m}\right. \\
& \left.\quad-6(n-m+1) a_{n-m+1}\right) a_{k+1} a_{m-k+1}
\end{aligned}
$$

$$
\begin{aligned}
& +\sum_{m=0}^{n} \sum_{k=0}^{n-m}\left(( m + 1 ) \left(m a_{m+1}+(\delta-\alpha \lambda)(m+2) a_{m+2}\right.\right. \\
& \left.-2 \beta a_{m+1}-M^{2} a_{m+1}\right) a_{k} a_{n-m-k} \\
& \left.\left.-(k+1)\left(6(m+1)(m+2) a_{m+2}+2(n-m-k) a_{m}\right) a_{k+1} a_{n-m-k}\right)\right),
\end{aligned}
$$

for $n=1,2,3, \ldots$. Notice from (11) that all the coefficients $a_{n}$ have been obtained in terms of $\beta, M$ and two unknowns $a_{2}$ and $\delta$. This constant $a_{2}$ remains unknown because of an end condition in (8). This unknown $a_{2}$ which characterizes the coefficient of skin friction, must be found in such a way that the derivative condition at far distance is satisfied. This is equivalent to determine the value of either $a_{2}$ of series (10) or $f^{\prime \prime}(0)$ of the system (1) and (2) because these are intrinsically related to each other through Eq. (6) as

$a_{2}=\frac{-1}{2(\alpha(1+\lambda)-\delta)}\left(f^{\prime \prime}(0)-\frac{2(1+\lambda)^{2}}{(\alpha(1+\lambda)-\delta)}\right)$.

Also, note that the constant $\delta$ defined in (4) is only for $\beta=-1$, but for other values of $\beta$ and $M$, it also needs to be determined. Thus, we have a two-parameter family of solutions to the Falkner-Skan equation. To determine one of these unknown constants $a_{2}$ or $f^{\prime \prime}(0)$ and $\delta$, we patch the series expansion of the closed form solution (9) with that of series (10) with $\beta=-1$ and $M=0$, which gives the same constant

$\delta=\alpha \lambda \pm \sqrt{-2(1+\lambda)+\alpha^{2}}$.

Thus, the above constant $\delta$ plays a crucial role in the present analysis. In fact, an exact solution of the MHD Falkner-Skan equation exists when the values of arguments in the square root function is positive. As mentioned earlier, there are different solutions to the problem depending on whether $\delta$ takes positive or negative sign before the square root function. We call it as the upper branch solution if it is positive, and the lower branch solution if it is negative. Fig. 1(a) and (b) shows the contour plots for these solution branches, wherein we divide the solution domain into five regions according to the values of $\lambda$ and $\alpha$. The white areas represent no solution to the problem. Physical significance for these regions will be discussed later.

To determine other constant $f^{\prime \prime}(0)$ or $a_{2}$, integrating the MHD Falkner-Skan Eq. (1) over the flow domain using the boundary conditions (2), we get

$$
\begin{gathered}
\int_{0}^{\infty}\left(f^{\prime}(\eta)-f^{\prime 2}(\eta)\right) d \eta+\beta \int_{0}^{\infty}\left(1-f^{\prime 2}(\eta)\right) d \eta \\
-M^{2} \int_{0}^{\infty}\left(f^{\prime}(\eta)-1\right) d \eta=f^{\prime \prime}(0)-\alpha(1+\lambda) .
\end{gathered}
$$

Since, the left hand side of (14) also involves $f^{\prime \prime}(0)$, it can be solved iteratively with suitable initial approximation for it. However, in order to effectively illustrate the method used to find $f^{\prime \prime}(0)$, we rewrite the above integral relation as

$$
\begin{array}{r}
\int_{0}^{\eta_{\max }}\left(f^{\prime}(\eta)-f^{\prime 2}(\eta)\right) d \eta+\beta \int_{0}^{\eta_{\max }}\left(1-f^{\prime 2}(\eta)\right) d \eta \\
-M^{2} \int_{0}^{\eta_{\max }}\left(f^{\prime}(\eta)-1\right) d \eta=f^{\prime \prime}(0)-\alpha(1+\lambda),
\end{array}
$$

where $\eta_{\max }=\lim \eta \rightarrow \infty$. Since, $f^{\prime \prime}(0)$ appears on both sides of the above relation, it has to be solved iteratively for all involved parameters. The solution of the above asymptotic integral relation is too complicated by the fact that the boundary condition is specified at infinity. Thus, the skin friction value $f^{\prime \prime}(0)$ could be found such that the end condition is satisfied. Therefore, in our computations, 'infinity' is numerically approximated by the large value of independent variable (i.e. $\eta_{\max }$ ). There is no such priori general method for estimating this value. Giving too small numerical 

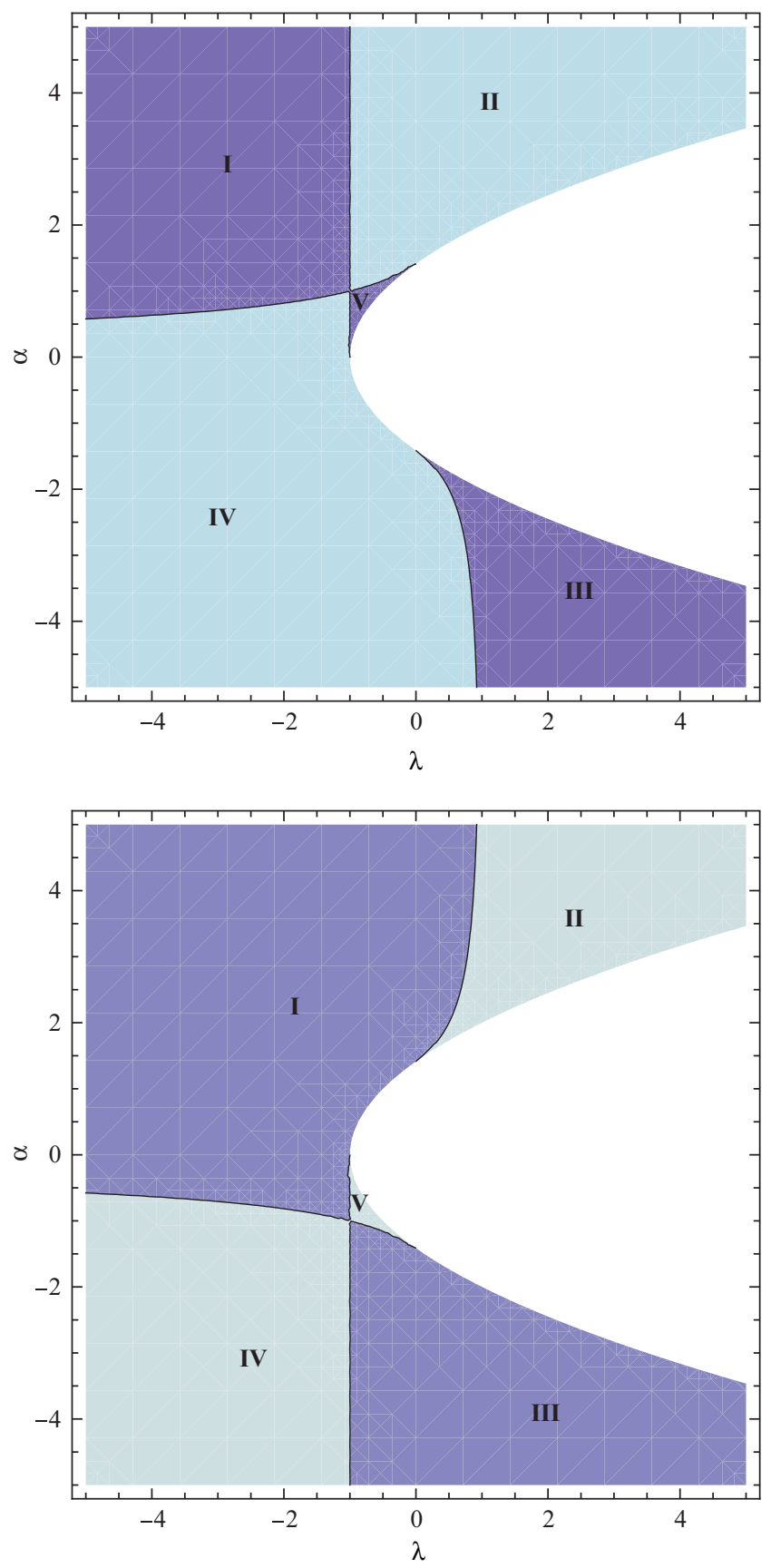

Fig. 1. Existence of solution for different values of $\alpha$ and $-\lambda$ for both upper (top) and lower (bottom) branch solutions.

value for $\eta_{\max }$ would not assure a uniformly valid convergent solution. And selecting too large value for $\eta_{\max }$ results either in asymptotic divergent series or in slow convergence of the series to satisfy derivative boundary condition at infinity. Moreover, when an arbitrary initial estimate is made, the successive approximations may not converge to the correct result. Hence, a method must be modified to logically estimate the value of $\eta_{\max }$ to get $f^{\prime \prime}(0)$ up to required accuracy. In the integration, the initial approximation of $f^{\prime \prime}(0)=2.56488$ is taken from exact analytical solution of the Falkner-Skan equation for $\beta=-1$ and $M=0$ for all other parameter $\beta$ and $M$ which serves a good initial estimate for $f^{\prime \prime}(0)$, and also it ensures the fast convergence. For small value of $\eta_{\max }$, the series is well behaved and can be integrated. So, in the process, once $f^{\prime \prime}(0)$ is assumed and the Pade' approximants which extend the region of validity of convergence, are used to sum the series, the integral relation can be integrated without any difficulty to determine a nearly correct value of $f^{\prime \prime}(0)$. With fewer iterations, $f^{\prime \prime}(0)$ can be obtained up to any desired accuracy. All of our simulations indicate that form (6) through series (10) gives convergent solution via Pade' approximants. More about Pade' approximants will be discussed later in the paper. Moreover, when $f^{\prime \prime}(0)$ is accurate enough, then corresponding velocity profiles approach their asymptotic value 1 . The results thus obtained for various values of $\beta$ and $M$ by the present method are seen to agree with those produced by the DNS of the MHD Falkner-Skan equation.

Therefore, we have obtained an exact solution of the MHD Falkner-Skan system (1) and (2) for all values of $\beta$ and $M$ in the form

$f(\eta)=\delta-\alpha \lambda+\eta-\frac{\delta-\alpha(1+\lambda)}{G(\eta)}$,

where the series $G(\eta)$ is given by (10).

To assess the efficiency of the present exact method, the results for skin friction value $f^{\prime \prime}(0)$ are compared with those produced by the direct numerical solution of the MHD Falkner-Skan equation. Table $1 \mathrm{a}$ and $\mathrm{b}$ presents the results for skin friction $f^{\prime \prime}(0)$ for different values of flow parameters. It can be seen that the results obtained by the present method are in excellent agreement with those given by the DNS for all values taken for comparison. Furthermore, these tables illustrate that the absolute value of skin friction increases for increasing pressure gradient parameter $\beta$ for all values of $M$ which signifies the presence of a magnetic field. Also, it increases for increasing suction and injection parameter $\alpha$. Thus, this can be concluded that the family of solutions though $f^{\prime \prime}(0)<0$ whose first-order derivative seems to approach to 1 exponentially.

Again, we compute the residual error of the MHD FalknerSkan equation for two sets of values at $\eta=1$ and $\eta=2$ for increasing number of terms in Pade' approximant series (i.e. $p=q=N \rightarrow \infty)$. This has been shown in Table 3a. It is clearly observed that the residual error approaches zero as $N \rightarrow \infty$ which confirms that solution (16) converges to the true solution. Table $3 \mathrm{~b}$ also confirms that above results wherein we compute solution (16) at different orders in Pade' approximants. This is calculated for $\beta=10, \lambda=-1.1, \alpha=0.5$, and for different values of magnetic parameter $M$. This shows that solution $f(\eta)$ remains constant for increasing $[N]$.

Now we describe the nature of the upper and lower branch solutions in Fig. 1 that make the velocity profiles greatly distinct from each other. Note that no solutions exist for the white area. Velocity profiles in each region in Fig. 1 behave differently for both solution branches. We chose the parameters from each of these regions. In Fig. 2, we plot the velocity profiles as a function of $\eta$ for accelerated flow $\beta=1$ and $M=1.0$ for upper branch solution (left column) in all the regions. Each region represents a typical velocity profiles behaving according to physical nature of the problem. In regions I and IV, the velocity curves are monotonically decreasing but satisfy their end condition, and the curve in regions II and III increases monotonically to its end boundary. However, in region I (II), the decrease (increase) rate is faster compared to other corresponding regions. However, we found a different velocity overshoot profile in region $\mathrm{V}$. The curve in region $\mathrm{V}$, the velocity first increases gradually to its peak value, then decreases and eventually satisfies its downstream condition. The similar results are also shown by Fang and Zhang [12] where their analysis was given only for $\beta=-1$ in the absence of magnetic field $(M=0)$. The similar trend can be seen in right column of Fig. 2 wherein we have shown velocity profiles for the lower branch solutions.

The following figures provide more physical insights mentioned below. Fig. 3 shows the variation of velocity profiles as a function of $\eta$ for different values of stretching parameter $\lambda$ for three sets of suction and injection parameter $\alpha$ and for two values 
Table 1

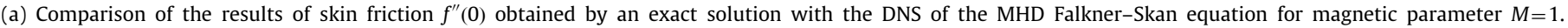
(b) Comparison of the results of skin friction $f^{\prime \prime}(0)$ obtained by an exact solution with the DNS of the MHD Falkner-Skan equation for magnetic parameter $M=2$.

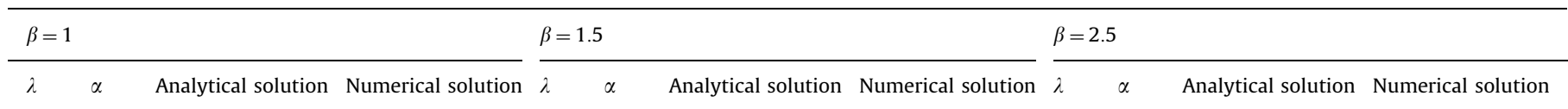

(a)

\begin{tabular}{cccc}
$(\mathrm{a})$ & & & \\
\hline-1 & & & \\
\hline-1.1 & -1.0 & -0.1439 & -0.14381 \\
& -0.5 & -0.1693 & -0.16561 \\
& 0 & -0.1904 & -0.19067 \\
& 0.5 & -0.2184 & -0.21891 \\
& 1 & -0.2502 & -0.2501 \\
-1.5 & -1.0 & -0.7729 & -0.77073 \\
& -0.5 & -0.8701 & -0.88027 \\
& 0 & -1.0168 & -1.00518 \\
& 0.5 & -1.1452 & -1.14521 \\
& 1 & -1.2991 & -1.29941 \\
-2.0 & -1.0 & -1.6672 & -1.66332 \\
& -0.5 & -1.8822 & -1.88347 \\
& 0 & -2.1383 & -2.13264 \\
& 0.5 & -2.41062 & -2.41029 \\
& 1 & -2.71491 & -2.71496
\end{tabular}

$\begin{array}{ccll}-1.1 & -1.0 & -0.16711 & -0.16906 \\ & -0.5 & -0.1913 & -0.19103 \\ & 0 & -0.2129 & -0.2159 \\ & 0.5 & -0.23536 & -0.24359 \\ -1.5 & 1 & -0.27191 & -0.27398 \\ & -1.0 & -0.90525 & -0.90646 \\ & 0.5 & -1.0162 & -1.01636 \\ & 0.5 & -1.1301 & -1.13988 \\ -2.0 & -1.0 & -1.4253 & -1.27691 \\ & -0.5 & -2.1716 & -1.42687 \\ & 0 & -2.4246 & -1.95654 \\ & 0.5 & -2.6875 & -2.1764 \\ & 1 & -2.9884 & -2.42197 \\ & & & -2.69311 \\ & & & -2.98891\end{array}$

$\begin{array}{ccc}-1.1 & -1.0 & -0.2112 \\ & -0.5 & -0.2344 \\ & 0 & -0.2591 \\ 0.5 & -0.2857 \\ & 1 & -0.3152 \\ -1.5 & -1.0 & -1.1377 \\ & -0.5 & -1.2488 \\ & 0 & -1.3711 \\ & 0.5 & -1.5102 \\ & 1 & -1.64901 \\ -2.0 & -1.0 & -2.4556 \\ & -0.5 & -2.6766 \\ 0 & -2.9111 \\ 0.5 & -3.1863 \\ 1 & -3.46609\end{array}$

$-0.21229$

$-0.23455$

$-0.25921$

$-0.28626$

$-0.31562$

$-1.13812$

$-1.24885$

$-1.37097$

$-1.50447$

$-1.64908$

$-2.45581$

$-2.67622$

$-2.91805$

$-3.18151$

$-3.46616$

(b)

$M=2$

\begin{tabular}{cccc}
\hline-1.1 & -1 & -0.21028 & -0.21026 \\
& -0.5 & -0.23189 & -0.23257 \\
& 0 & -0.2572 & -0.25729 \\
& 0.5 & -0.28327 & -0.28442 \\
-1.5 & 1 & -0.30691 & -0.31387 \\
& -1 & -1.08893 & -1.08974 \\
& 0.5 & -1.20159 & -1.20158 \\
& 0.5 & -1.32416 & -1.32514 \\
& 1 & -1.46031 & -1.46035 \\
-2.0 & -1 & -2.21052 & -1.60688 \\
& -0.5 & -2.49779 & -2.20252 \\
& 0 & -2.74401 & -2.49686 \\
& 0.5 & -3.01035 & -3.74384 \\
& 1 & -3.30386 & -3.30472
\end{tabular}

$\begin{array}{cccl}-1.1 & -1 & -0.22658 & -0.22951 \\ & -0.5 & -0.24908 & -0.25192 \\ & 0 & -0.27570 & -0.27659 \\ & 0.5 & -0.30527 & -0.3035 \\ & 1 & -0.33436 & -0.33259 \\ -1.5 & -1 & -1.19548 & -1.19553 \\ & -0.5 & -1.30387 & -1.3076 \\ & 0 & -1.43089 & -1.43055 \\ & 0.5 & -1.5694 & -1.56437 \\ -2.0 & -1 & -1.70770 & -1.70877 \\ & -0.5 & -2.50649 & -2.50643 \\ & 0 & -2.73079 & -2.73056 \\ & 0.5 & -3.24156 & -2.97564 \\ & 1 & -3.52749 & -3.24166 \\ & & & \end{array}$

$\begin{array}{ll}-0.5 & -0.28711\end{array}$

$0 \quad-0.31164$

$0.5-0.33819$

$1-0.36682$

$\begin{array}{lll}-1.5 & -1 & -1.38644\end{array}$

$-0.5-1.4979$

$0 \quad-1.62116$

$0.5-1.75305$

$1-1.89432$

$-2.0-1 \quad-2.92549$

$-0.5-3.1502$

$0-3.39253$

$0.5-3.65308$

$1-3.9333$

$$
\begin{aligned}
& -0.26446 \\
& -0.28706 \\
& -0.31166 \\
& -0.33825 \\
& -0.3668 \\
& -1.38645 \\
& -1.49899 \\
& -1.62117 \\
& -1.753 \\
& -1.89432 \\
& -2.92589 \\
& -3.15009 \\
& -3.39277 \\
& -3.65398 \\
& -3.9335
\end{aligned}
$$

of magnetic parameter $M$. Note that though values of $\lambda$ are negative, the profiles start from the positive values because of the initial condition $f^{\prime}(0)=-\lambda$. The velocity curves for injection and suction are very different from an impermeable case $(\alpha=0)$. Presence of the parameter $\alpha$ makes the boundary layer flow greatly enriched. It is observed that fluid injection into the flow increases the boundary layer thickness, whereas it decreases for the suction parameter $\alpha$. The similar structure can be observed for other stretching parameter $\lambda$. Furthermore, when Hartmann number increases the boundary layer thickness decreases, see right side of Fig. 3. In Fig. 4, the similar observation can be made from the above analysis, wherein we investigate the effect of pressure gradient $\beta$ and Hartmann number $M$. The velocity profiles in this case decrease revealing the fact that the effect of $\beta$ is to decelerate the velocity of the fluid, and hence it reduces the momentum boundary layer thickness.

In Fig. 5, we investigate the variation of dimensionless velocity profiles with respect to the variation in the Hartmann number $M$ in the MHD boundary layer flow for different values of $\beta$. The momentum interacts with each other, and the profiles in figure have strong variations with a distance along the boundary layer. When the magnetic parameter increases, the velocity gradient decreases, clearly showing the effect of the magnetic field in the flow system. When the magnetic field is applied, the system acquires more magnetization, the momentum force decreases, as a result the flow moves slowly along the whole field. Also, the variation in the Lorentz force offers more resistance to the flow phenomena. Whereas the reverse trend is observed for smaller values of $M$. Thus, the application of the magnetic field on the boundary layer flow decreases the thickness of the boundary layer and this typical trend is observed for all values of $\beta$.

Thus, an application of magnetic field on the two-dimensional viscous boundary layer flow over a semi-infinite flat plate reveals an important flow characters. The flow problem has been solved both analytically and asymptotically in the large $\eta$ limit. It is found that the thickness of the boundary layer decreases for increasing Hartmann number $M$ and pressure gradient parameter $\beta$, while absolute of value of skin friction $f^{\prime \prime}(0)$ increases when they increase. All these observed phenomena are in accordance with dynamics of the problem.

\section{Asymptotic solution}

The derivative boundary condition at edge of the boundary layer suggests to look for local behavior i.e. $\left|f^{\prime}(\eta)-1\right| \ll 1$ as $\eta \rightarrow \infty$. In order to formulate the problem at the edge, we introduce a stream-function $E(\eta)$ such that

$f(\eta) \sim \alpha+\eta+E(\eta)$

where $E^{\prime}(\eta) \ll 1$. Also, it is instructive to compare the solutions of (17) with those of exact method presented in Section 3 for all values. Substituting (17) into the MHD Falkner-Skan system (1) and (2), and upon linearizing the resulting ordinary 

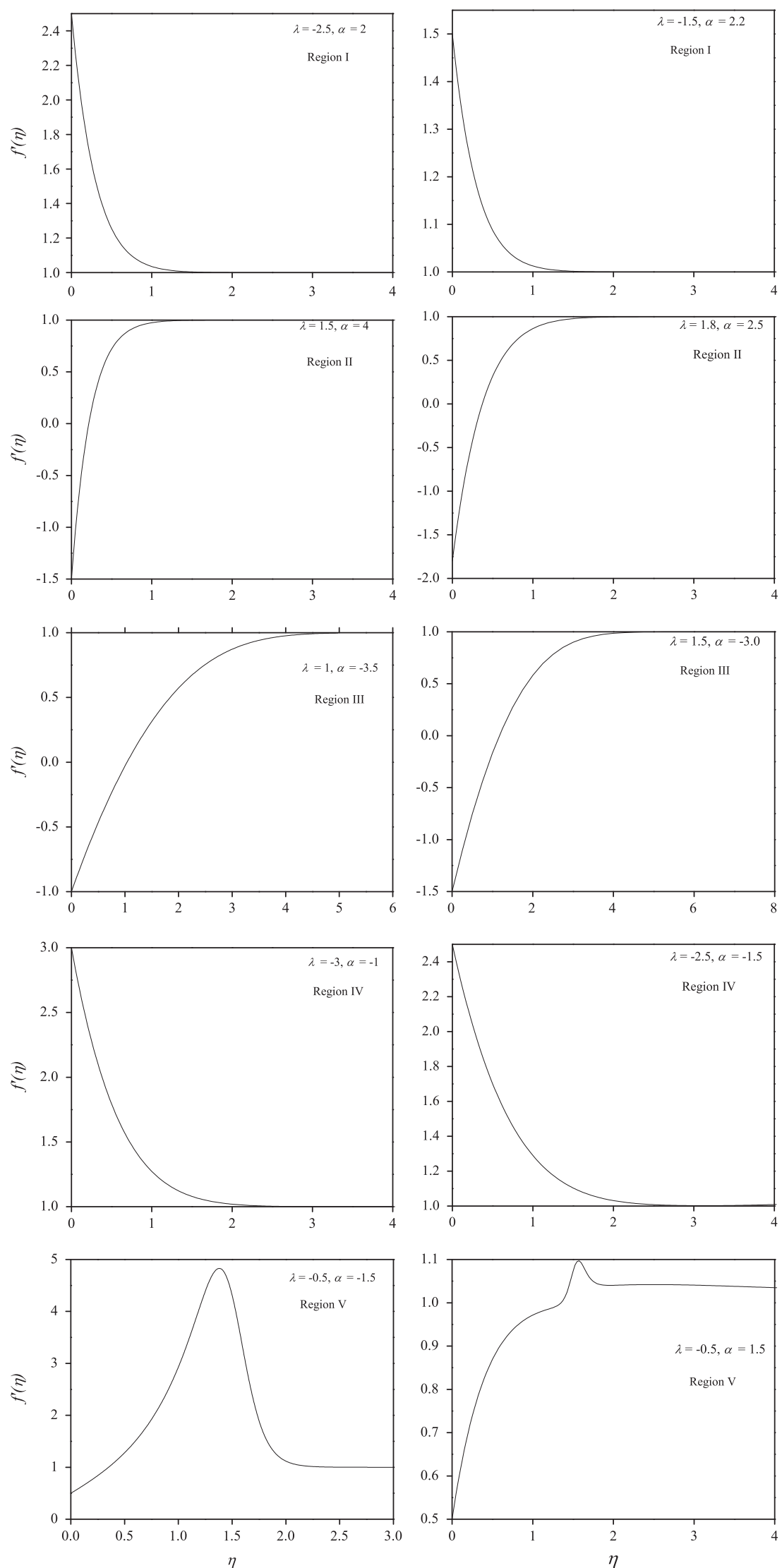

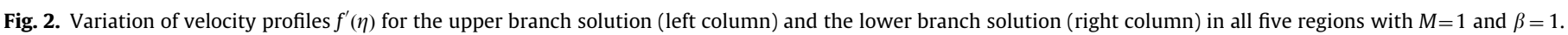



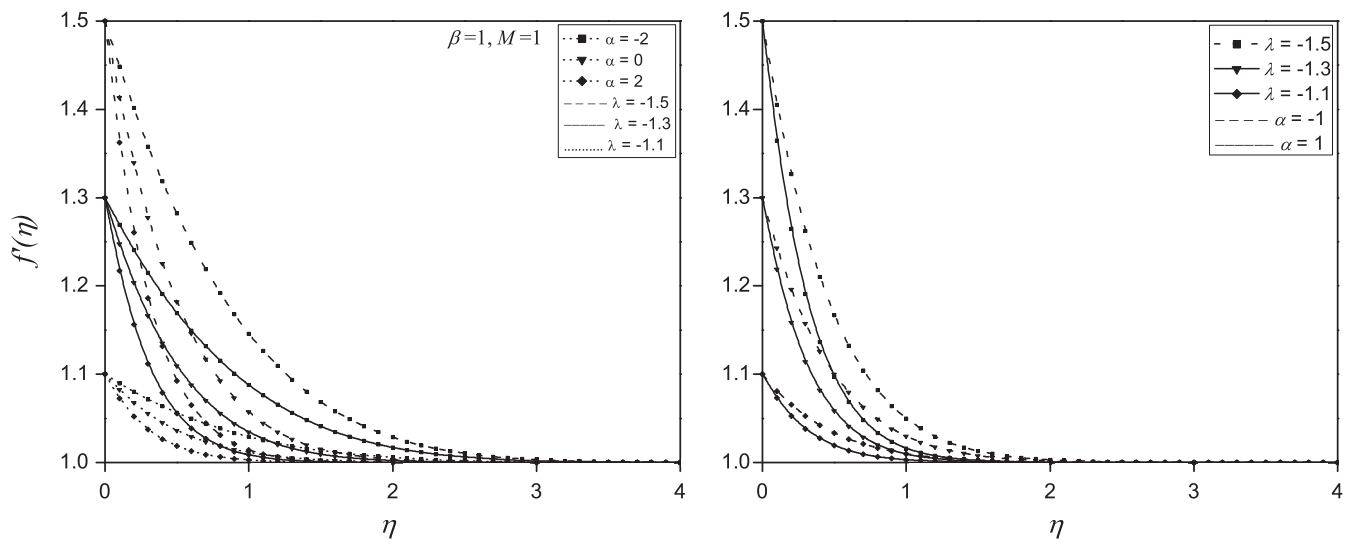

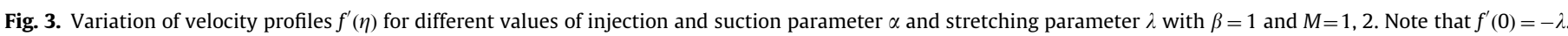
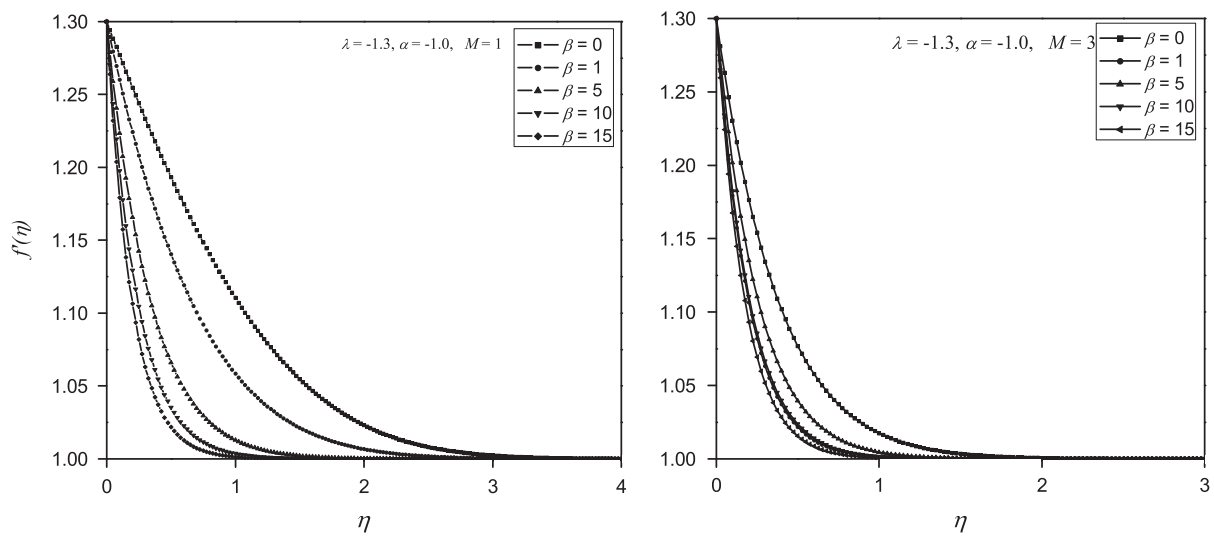

Fig. 4. Variation of velocity profiles $f^{\prime}(\eta)$ for different values of pressure gradient parameter $\beta$ with $\lambda=-1.3, \alpha=-1$ and $M=1,2$, and 3 . Note that $f^{\prime}(0)=-\lambda$.

differential equation, we get

$E^{\prime \prime \prime}(\eta)+(\alpha+\eta) E^{\prime \prime}(\eta)-\left(2 \beta+M^{2}\right) E^{\prime}(\eta)=0$,

and boundary conditions take the form

$E(0)=0, \quad E^{\prime}(0)=-(1+\lambda), \quad E^{\prime}(+\infty)=0$.

Solution of Eq. (18) subjected to the conditions (19) is given by

$E^{\prime}(\eta)=$

$\frac{-(1+\lambda) F\left(-\beta-\frac{M^{2}}{2}, \frac{1}{2},-\frac{(\eta+\alpha)^{2}}{2}\right)+(1+\lambda) C(\eta+\alpha) F\left(\frac{1-2 \beta-M^{2}}{2}, \frac{3}{2},-\frac{(\eta+\alpha)^{2}}{2}\right)}{F\left(-\beta-\frac{M^{2}}{2}, \frac{1}{2},-\frac{(\alpha)^{2}}{2}\right)-C \alpha F\left(\frac{1-2 \beta-M^{2}}{2}, \frac{3}{2},-\frac{(\alpha)^{2}}{2}\right)}$,

where $C=(\Gamma(1 / 2) / \Gamma(3 / 2))\left(\Gamma\left(1+\beta+M^{2} / 2\right) / \Gamma\left(1 / 2+\beta+M^{2} / 2\right)\right), \quad \Gamma$ is the Gamma function and $F(\tilde{a}, \tilde{b}, \eta)$ is the confluent hypergeometric function. Table $2 a$ and $b$ compare the values of skin friction $f^{\prime \prime}(0)$ obtained through (17) and (20) with those given by Eq. (16) for two values of Hartmann number $M$ for other different parameters. The results are quite remarkable. Behaviors of the solutions at far distance are entirely in accordance with physical phenomenon of the flow problem.

\section{Discussions and conclusions}

We have developed a new exact solution for the MHD Falkner-Skan Eq. (1) subject to the boundary conditions (2) for all values of the pressure gradient parameter $\beta$ and Hartmann number $M$. This new method utilizes and embeds the known exact solution of the problem for $\beta=-1$ and $M=0$, which is rewritten to give an exact solution for general values of $\beta$ and $M$. Function (16) through series (10) is an analytical solution. The validity and efficiency of the method has been checked for various values of $\beta$ and $M$, and compared with that of the DNS of the problem.

The present method can be compared to homotopy analysis method in the following way; it is well known that the latter method requires an appropriate 'initial guess' and operators to obtain a uniformly valid convergent solution to the problem in question, whereas the present method utilizes its own 'closedform solution' obtained for the particular parameters for obtaining analytical solution for other values of parameters which makes the method more analytically stronger than any other method. Thus, the present method is easy to use for non-linear boundary layer problems.

We now give a simple analysis for usage of Pade' approximants in summation of the series $G(\eta)$. That is to show if the infinite series $G(\eta)$ is convergent, then a function $f(\eta)$ is analytical solution of the MHD Falkner-Skan equation. The Pade' approximants perform an analytic continuation of the series outside its radius of convergence. Employing the classical Pade' technique to power series $G(\eta)$, we have

$G(\eta)=\sum_{n=0}^{\infty} a_{n} \eta^{n}=\frac{\sum_{k=0}^{p} b_{k} \eta^{k}}{\sum_{k=0}^{q} c_{k} \eta^{k}}$

where $c_{0}=1$ without loss of generality, and remaining $(p+q+1)$ coefficients $b_{0}, b_{1}, b_{2}, \ldots, b_{p}, c_{1}, c_{2}, c_{3}, \ldots, c_{q}$ are to be determined uniquely from the coefficients $a_{n}$, so that the first $(p+q+1)$ coefficients in the Taylor series expansion of the Pade' approximants 

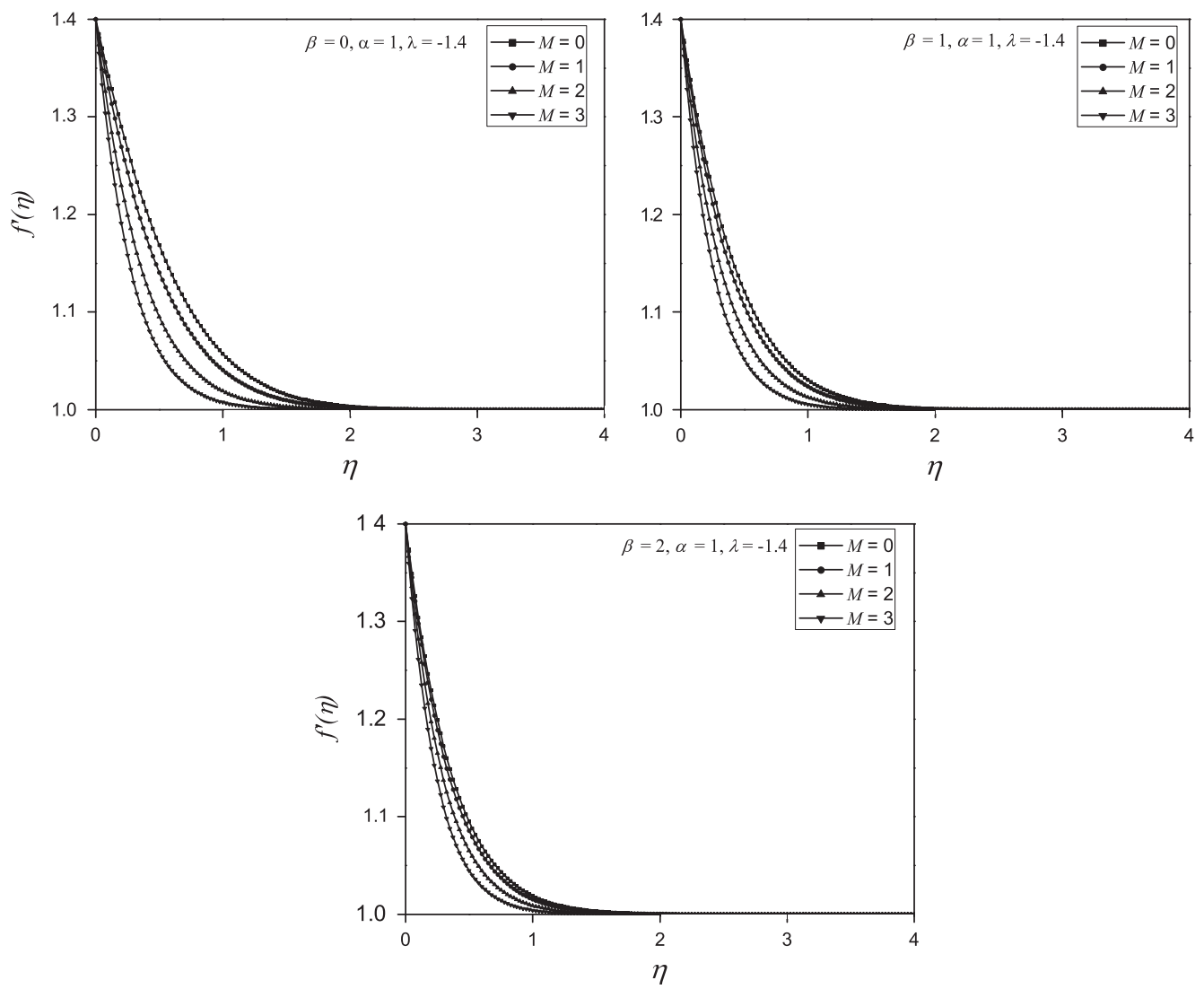

Fig. 5. Variation of velocity profiles $f^{\prime}(\eta)$ for different values of Hartmann number $M$ for $\beta=0,1,2, \alpha=1$ and $\lambda=-1.4$. Note that $f^{\prime}(0)=-\lambda$.

Table 2

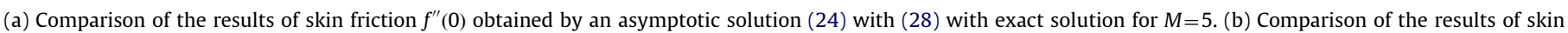
friction $f^{\prime \prime}(0)$ obtained by an asymptotic solution (24) with (28) with exact solution for $M=10$.

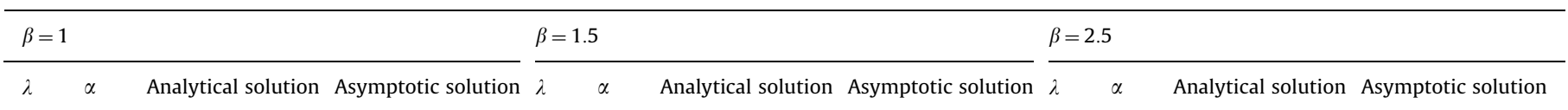

(a)

$M=5$

\begin{tabular}{|c|c|c|c|c|c|c|c|c|c|c|c|}
\hline \multirow[t]{4}{*}{-1.1} & -1 & -0.47728 & -0.47637 & -1.1 & -1 & -0.48708 & -0.4858 & -1.1 & -1 & -0.50588 & -0.50416 \\
\hline & -0.5 & -0.50077 & -0.49981 & & -0.5 & -0.51051 & -0.50926 & & -0.5 & -0.52921 & -0.52767 \\
\hline & 0.5 & -0.55131 & -0.55027 & & 0.5 & -0.56087 & -0.5597 & & 0.5 & -0.57948 & -0.57808 \\
\hline & 1 & -0.57774 & -0.57727 & & 1 & -0.58699 & -0.58666 & & 1 & -0.60741 & -0.60497 \\
\hline \multirow[t]{4}{*}{-1.5} & -1 & -2.40588 & -2.38187 & -1.5 & -1 & -2.46047 & -2.42898 & -1.5 & -1 & -2.56639 & -2.52078 \\
\hline & -0.5 & -2.52171 & -2.49908 & & -0.5 & -2.57743 & -2.5463 & & -0.5 & -2.68524 & -2.63833 \\
\hline & 0.5 & -2.76521 & -2.75135 & & 0.5 & -2.8348 & -2.79849 & & 0.5 & -2.93428 & -2.89037 \\
\hline & 1 & -2.91012 & -2.88637 & & 1 & -2.96518 & -2.93332 & & 1 & -3.0681 & -3.02484 \\
\hline \multirow{4}{*}{-2.0} & -1 & -4.85861 & -4.76374 & -2.0 & -1 & -4.98258 & -4.85795 & -2.0 & -1 & -5.22193 & -5.04156 \\
\hline & -0.5 & -5.09286 & -4.99816 & & -0.5 & -5.21564 & -5.09261 & & -0.5 & -5.45605 & -5.27666 \\
\hline & 0.5 & -5.59729 & -5.5027 & & 0.5 & -5.72884 & -5.59698 & & 0.5 & -5.95747 & -5.78075 \\
\hline & 1 & -5.86697 & -5.77275 & & 1 & -5.99859 & -5.86664 & & 1 & -6.22383 & -6.04969 \\
\hline
\end{tabular}

(b)

$M=10$

\begin{tabular}{|c|c|c|c|c|c|c|c|c|c|c|c|}
\hline \multirow[t]{4}{*}{-1.1} & -1 & -0.96396 & -0.96354 & -1.1 & -1 & -0.96922 & -0.96846 & -1.1 & -1 & -0.97855 & -0.97824 \\
\hline & -0.5 & -0.98877 & -0.98767 & & -0.5 & -0.99325 & -0.9926 & & -0.5 & -1.00336 & -1.00238 \\
\hline & 0.5 & -1.03807 & -1.0378 & & 0.5 & -1.04342 & -1.04272 & & 0.5 & -1.05345 & -1.0525 \\
\hline & 1 & -1.06406 & -1.06378 & & 1 & -1.06936 & -1.0687 & & 1 & -1.07944 & -1.07846 \\
\hline \multirow{4}{*}{-1.5} & -1 & -4.8413 & -4.81771 & -1.5 & -1 & -4.85873 & -4.84231 & -1.5 & -1 & -4.91566 & -4.89118 \\
\hline & -0.5 & -4.9485 & -4.93838 & & -0.5 & -4.9788 & -4.96301 & & -0.5 & -5.03636 & -5.01191 \\
\hline & 0.5 & -5.20162 & -5.18899 & & 0.5 & -5.22988 & -5.21361 & & 0.5 & -5.28674 & -5.26251 \\
\hline & 1 & -5.33415 & -5.31892 & & 1 & -5.3595 & -5.3435 & & 1 & -5.41644 & -5.39232 \\
\hline \multirow[t]{4}{*}{-2.0} & -1 & -9.68455 & -9.63541 & -2.0 & -1 & -9.75018 & -9.68463 & -2.0 & -1 & -9.87995 & -9.78236 \\
\hline & -0.5 & -9.92459 & -9.87677 & & -0.5 & -9.99146 & -9.92602 & & -0.5 & -10.1206 & -10.0238 \\
\hline & 0.5 & -10.4217 & -10.378 & & 0.5 & -10.4933 & -10.4272 & & 0.5 & -10.6216 & -10.525 \\
\hline & 1 & -10.6731 & -10.6379 & & 1 & -10.7526 & -10.687 & & 1 & -10.8807 & -10.7846 \\
\hline
\end{tabular}


Table 3

(a) Decaying residual error of the MHD Falkner-Skan Eqs. (1) and (2) for $\lambda=-1.1$ and $\alpha=0.5$. (b) Comparison of solution (16) of the MHD Falkner-Skan Eqs. (1) and (2) for $\beta=10$ at different orders of Pade' approximants.

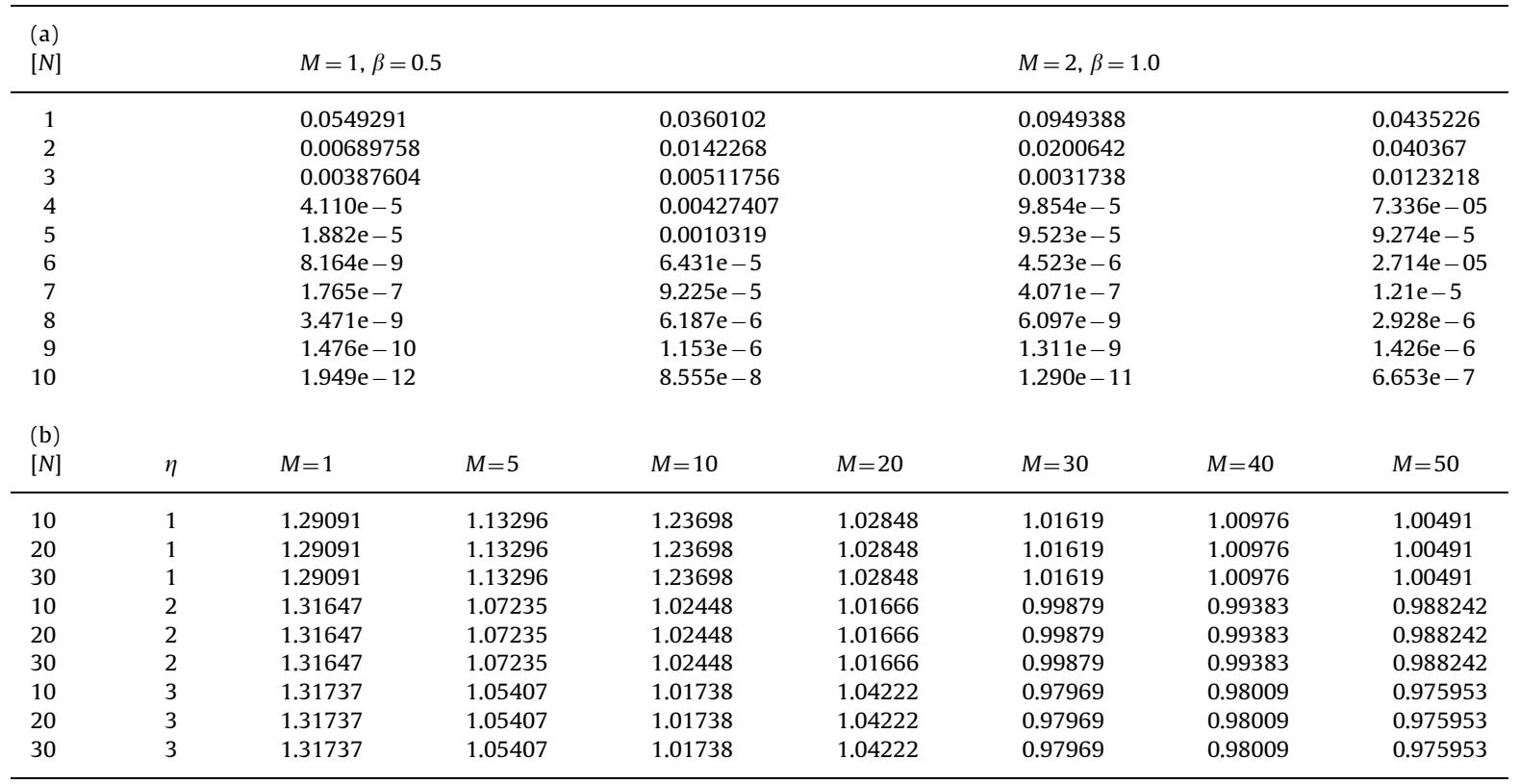

should match the first $(p+q+1)$ coefficients in the infinite series $G(\eta)$. Therefore, from solution (16), we have

$$
\begin{aligned}
f(\eta) & =\delta-\alpha \lambda+\eta-\frac{\delta-\alpha(1+\lambda)}{\sum_{n=0}^{\infty} a_{n} \eta^{n}} \\
& =\delta-\alpha \lambda+\eta-\frac{(\delta-\alpha(1+\lambda)) \sum_{k=0}^{q} c_{k} \eta^{k}}{\sum_{k=0}^{p} b_{k} \eta^{k}}=\psi_{p, q}(\eta),
\end{aligned}
$$

where conveniently written function $\psi_{p, q}(\eta)$ is the Pade' approximants to analytical solution $f(\eta)$ through the infinite series $G(\eta)$. Note that using the elementary arithmetic operations, the coefficients $(p+q+1)$ are easy to evaluate numerically. It immediately follows from (22) that

$f^{\prime}(\eta)=\psi_{p, q}^{\prime}(\eta)$,

$f^{\prime \prime}(\eta)=\psi_{p, q}^{\prime \prime}(\eta)$

$f^{\prime \prime \prime}(\eta)=\psi_{p, q}^{\prime \prime \prime}(\eta)$, etc.

Therefore, from system (1), we obtain

$$
\begin{aligned}
\frac{d^{3} \psi_{p, q}(\eta)}{d \eta^{3}} & +\psi_{p, q}(\eta) \frac{d^{2} \psi_{p, q}(\eta)}{d \eta^{2}} \\
& +\beta\left(1-\left(\frac{d \psi_{p, q}(\eta)}{d \eta}\right)^{2}\right)-M^{2}\left(\frac{d \psi_{p, q}(\eta)}{d \eta}-1\right)=0 .
\end{aligned}
$$

Note that if the infinite series (10) is convergent, then the solution $f(\eta)=\psi_{p, q}(\eta)$ must be a solution of Eq. (23) in the sense that Eq. (1) uniquely satisfies. We emphasize here that the Pade' approximants converge to the true solution as $p$ and $q$ tend to infinity. It is easy to calculate the coefficients $a_{n}$ using Mathematica and Maple, and also easy to code with MATLAB. Also, it is worth to mention that the function $\psi_{p, q}$ embeds the known closed-form solution (9) of system (7) and (8) for $\beta=-1$ and $M=0$ in its base function which assures that the convergence is guaranteed. A close examination and careful evaluation of the coefficients $\left(d_{n}\right)$ in the new stream-function $\psi_{p, q}(\eta)(=f(\eta))$ suggest that the more accurate estimation of the region of convergence can be obtained using the Domb-Sykes plot. The Domb-Sykes plots for two-sets of flow quantities have been shown in Fig. 6. This plot is typical for the analysis of the series in that
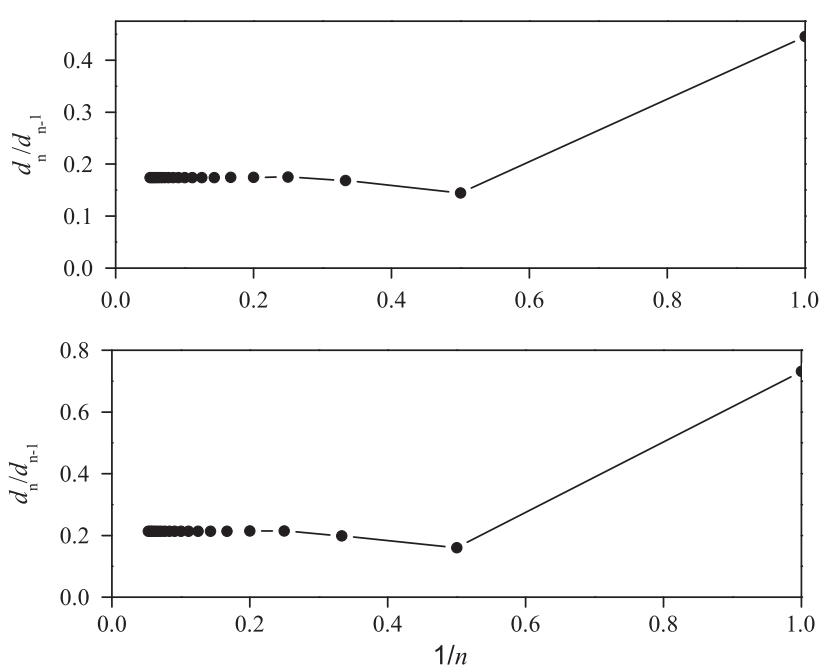

Fig. 6. Domb-Sykes plot for the new stream-function $\psi$.

the curves show an establishment of a linear relationship between the ratio of coefficients as the number of terms increases $(>2)$. This linear curve easily helps to extrapolate the ratios $d_{n} / d_{n-1}$ to $1 / n=0$ which gives the intercept value of 0.179 in the graph above, and 0.202 in the second graph. Thus, the radius of convergence of the function in the first curve is $\frac{1}{0.179} \sim 5.6$, and for the other curve it is approximately 5 . Also, the curves in all figures show that the end condition is satisfied much earlier than these values which affirm the analyticity of the method. These suggest that the results obtained by our method by setting convergent range agree with the range given by numerical solution. Therefore, all of our computations indicate that series (10) is convergent implying the solution $f(\eta)$ is a complete analytical solution of the MHD FalknerSkan equation for all parameters under investigation.

Note also that function (16) is an analytical solution of the MHD Falkner-Skan equation, the constant $\delta$ restricts us to analyze for complete set of the parameters (see the arguments in square root function, i.e. $\left.-2(1+\lambda)+\alpha^{2}<0\right)$. The closed form 
solution (4) of (1) exists only when the arguments in square root in $\delta$ are positive, so is true for all values of $\beta$ and $M$. Therefore, the method has to be modified such that solution (16) is an exact solution of the problem, and a serious investigation is underway. Hopefully this can be achieved through when we follow analysis of Yang and Chein [14] (with $\lambda=0$ ) where they give a closed form solution for $\beta=-1$ in terms of confluent hypergeometric functions of the first kind.

\section{Acknowledgments}

This work was supported by the University Grants Commission, New Delhi, India, under major research project Grant no. 39-32/2010 (S.R.). Authors would like to thank referees for the valuable comments which enhanced the quality of the paper.

\section{Appendix}

Consider the MHD two-dimensional viscous and incompressible boundary layer flow over a semi-infinite flat plate moving with a constant velocity $U_{w}$ in an electrically conducting fluid in the presence of applied magnetic field $B(x)$. The $x$-axis is measured along the direction of the flow, and $y$-axis is normal to it. The moving plate is considered to have permeable with lateral mass flux velocity $V_{w}$. The magnetic field is applied transversely to the boundary layer flow. The Reynolds number is too small so that the induced magnetic field is negligible, also strength of electric field due to polarization of charges is negligible. Finally the free-stream velocity is $U(x)$. Under these approximations, the governing boundary layer equations are given by

$\frac{\partial u}{\partial x}+\frac{\partial v}{\partial y}=0$

$u \frac{\partial u}{\partial x}+v \frac{\partial u}{\partial y}=U(x) \frac{\partial U(x)}{\partial x}+v \frac{\partial^{2} u}{\partial y^{2}}-\frac{\sigma B^{2}(x)}{\rho}(u-U(x))$,

where $u$ and $v$ are the velocity components in the $x$ and $y$ directions, $v$ is the kinematic viscosity of the fluid, $\sigma$ is the electrical conductivity, $\rho$ is the fluid density, and the magnetic field is given by $B(x)=B_{0} x^{(m-1) / 2}, U(x)$ is the velocity at the edge of the boundary layer which obeys the power law relation $U(x)=U_{\infty} x^{m}$, where $x$ is the distance measured from the onset of the boundary layer, $U_{\infty}$ and $m$ are constants. The relevant boundary conditions for the above model are

at $y=0: \quad u=U_{w}(x), \quad v=V_{w}(x), \quad$ and

as $\frac{y}{\delta} \rightarrow \infty: \quad u \rightarrow U(x)$,

where $U_{w}(x)$ is the stretching surface velocity which obeys the powerlaw relation $U_{w}(x)=U_{0 w} x^{m}$. Using the following similarity transformations:

$\psi=\sqrt{\frac{2 v x U(x)}{1+m}} f(\eta), \quad \eta=\sqrt{\frac{(1+m) U(x)}{2 v x}} y, \quad \beta=\frac{2 m}{1+m}$,

where the stream-function $\psi(x, y)$ is defined as

$(u, v)=\left(\frac{\partial \psi}{\partial y},-\frac{\partial \psi}{\partial x}\right)$,

into the above non-linear system (24) and (25), we get a third order ordinary differential equation

$f^{\prime \prime \prime}(\eta)+f(\eta) f^{\prime \prime}(\eta)+\beta\left(1-f^{\prime 2}(\eta)\right)-M^{2}\left(f^{\prime}(\eta)-1\right)=0, \quad '=\frac{d}{d \eta}$,

with the boundary conditions

$f(0)=\alpha, \quad f^{\prime}(0)=-\lambda, \quad f^{\prime}(+\infty)=1$, which is the MHD Falkner-Skan boundary layer equation. Here $f(\eta)$ is the non-dimensional stream-function, and $\eta$ is a new similarity variable, $\alpha\left(=-\sqrt{2 x /(m+1) v U(x)} V_{w}(x)\right)$ is the suction or injection parameter, $\alpha>0$ represents suction and $\alpha<0$ is the injection, and whereas $\alpha=0$ is impermeable of the plate. $\lambda\left(=-U_{w} / U_{\infty}\right)$ is the stretch of the boundary, $\lambda>0$ and $\lambda<0$ correspond to moving plate in opposite and in the same direction to the free-stream velocity, whereas $\lambda=0$ is the case for fixed plate, $\beta$ is the pressure gradient parameter, $\beta>0$ is the favorable, and $\beta<0$ is the adverse pressure gradient, whereas $\beta=0$ is the two-dimensional Blasius flow over a flat plate. Parameter $M$ is the magnetic (Hartmann number) parameter which is the ratio of electromagnetic force to the viscous force. For $\beta=0$ and $M=0$, the above problem reduces to the Blasius flow that describes a two-dimensional flow over a flat plate with mass transfer and stretch of the plate, and is studied by several investigators with different cases and hence no comment is needed.

\section{References}

[1] K.R. Rajagopal, A.S. Gupta, T.Y. Na, A note on the Falkner-Skan flows of a nonnewtonian fluid, International Journal of Non-Linear Mechanics 18 (1983) 313-320.

[2] C.H. Hsu, C.H. Chen, J.T. Teng, Temperature and flow fields for the flow of a second grade fluid past a wedge, International Journal of Non-Linear Mechanics 32 (1997) 933-946.

[3] D.O. Olagunju, The Falkner-Skan flow of a viscoelastic fluid, International Journal of Non-Linear Mechanics 41 (2006) 825-829.

[4] M. Anabtawi, S. Khuri, On the generalized Falkner-Skan equation governing boundary layer flow of a FENE-P fluid, Applied Mathematics Letters 20 (2007) 1211-1215.

[5] M.R. Vera, A. Valencia, Solutions of Falkner-Skan equation with heat transfer by Fourier series, International Communications in Heat and Mass Transfer 37 (2010) 761-765.

[6] D.R. Hartree, On an equation occurring in Falkner-Skan approximate treatment of the equations of the boundary layer, Proceedings of the Cambridge Philosophical Society 33 (1937) 223-239.

[7] K. Stewartson, Further solutions of the Falkner-Skan equation, Proceedings of the Cambridge Philosophical Society 50 (1954) 454-465.

[8] P.A. Libby, T.M. Liu, Further solutions of the Falkner-Skan equation, AIAA Journal 5 (1967) 1040-1042.

[9] N. Riley, P.D. Weidman, Multiple solutions of the Falkner-Skan equation for flow past a stretching boundary, SIAM Journal on Applied Mathematics 49 (1989) 1350-1358.

[10] S.J. Liao, Uniformly valid analytic solution of two-dimensional viscous flow over a semi-infinite flat plate, The Journal of Fluid Mechanics 385 (1999) $101-128$

[11] N. Afzal, V.K. Luthra, Highly accelerated laminar flow at moderately large Reynolds number, AIAA Journal 12 (1975) 529-532.

[12] T. Fang, J. Zhang, An exact analytical solution of the Falkner-Skan equation with mass transfer and wall stretching, International Journal of Non-Linear Mechanics 43 (2008) 1000-1006.

[13] N. Afzal, Falkner-Skan equation for flow past a stretching surface with suction or blowing: analytical solutions, Applied Mathematics and Computation 217 (2010) 2724-2736.

[14] H.T. Yang, L.C. Chien, Analytic solution of the Falkner-Skan equation when $\beta=-1$ and $\gamma=0$, SIAM Journal on Applied Mathematics 29 (1975) 558-569.

[15] B. Yao, Approximate analytical solution to the Falkner-Skan wedge flow with the permeable wall of uniform suction, Communications in Nonlinear Science and Numerical Simulation 14 (2009) 3320-3326.

[16] B. Yao, J. Chen, Series solution to the Falkner-Skan equation with stretching boundary, Applied Mathematics and Computation 208 (2009) 156-164.

[17] B. Raftari, K. Vajravelu, Homotopy analysis method for MHD viscoelastic fluid flow and heat transfer in a channel with a stretching wall, Communications in Nonlinear Science and Numerical Simulation 17 (2012) 4149-4162.

[18] P.L. Sachdev, Nonlinear Diffusive Waves, CUP, Cambridge, 1987.

[19] P.L. Sachdev, K.R.C. Nair, V.G. Tikekar, Generalized Burgers equations and Euler-Painleve trancedents I, Journal of Mathematical Physics 27 (1986) 1506-1523.

[20] P.L. Sachdev, K.R.C. Nair, V.G. Tikekar, Generalized Burgers equations and Euler-Painlevé transcendents III, Journal of Mathematical Physics 29 (1988) 2397-2404.

[21] P.L. Sachdev, R.B. Kudenatti, N.M. Bujurke, Exact analytic solution of a boundary value problem for the Falkner-Skan equation, Studies in Applied Mathematics 120 (2008) 1-16.

[22] R.B. Kudenatti, A new exact solution for boundary layer flow over a stretching plate, International Journal of Non-Linear Mechanics 47 (2012) 727-733.

[23] M. Rashidi, E. Erfani, A new analytical study of MHD stagnation-point flow in porous media with heat transfer, Computers \& Fluids 40 (2011) 172-178. 